\title{
Characterizations of the dilation of frame generator dual pairs for group
}

Liang $\mathrm{Li}^{i^{*}}$ and Pengtong $\mathrm{Li}^{1}$

\begin{abstract}
In this paper, we are interested in the dilation problem on frame generator dual pairs for a unitary representation in Hilbert spaces. We show the existence of a Riesz generator dilation dual pair of a frame generator dual pair in Hilbert spaces. Then we reveal the uniqueness of such dilations in the sense of similarity and give a characterization of the dilation of frame generator alternate dual pairs by that of the canonical dual pair in terms of a special operator. We also exhibit that the corresponding operator between two dilations of a frame generator dual pair is in a special structure.
\end{abstract}

MSC: 46L10; 42C40; 42C15

Keywords: Dilations; Group representations; Frame generators; Dual frame generators; Riesz generators

\section{Introduction}

In [12], Sun initiated the concept of g-frame as follows. Let $\mathbb{J}$ be a finite or countable index set. Let $A_{i} \in B\left(H, H_{i}\right)$, where $H_{i}$ is a separable Hilbert space for any $i \in \mathbb{J}$. If there exist two constants $a, b$ such that

$$
a\|f\|^{2} \leq \sum_{i \in \mathbb{J}}\left\|A_{i} f\right\|^{2} \leq b\|f\|^{2}, \quad \forall f \in H,
$$

we call $\left\{A_{i}\right\}_{i \in \mathbb{J}}$ a $g$-frame for $H$. $\left\{A_{i}\right\}_{i \in \mathbb{J}}$ is called a tight $g$-frame for $H$ if $a=b$. Specially, if $a=b=1$, we say that $\left\{A_{i}\right\}_{i \in \mathbb{J}}$ is a Parseval $g$-frame for $H$. If only the right-hand inequality holds, then $\left\{A_{i}\right\}_{i \in \mathbb{J}}$ is called a $g$-Bessel sequence for $H$. If $\overline{\operatorname{span}}\left\{A_{i}^{*} H_{i}\right\}_{i \in \mathbb{J}}=H$, we say that $\left\{A_{i}\right\}_{i \in \mathbb{J}}$ is $g$-complete in $H$. If $\left\{A_{i}\right\}_{i \in \mathbb{J}}$ is g-complete such that

$$
a\left\|\left\{g_{i}\right\}_{i \in \mathbb{J}}\right\|^{2} \leq\left\|\sum_{i \in \mathbb{J}} A_{i}^{*} g_{i}\right\|^{2} \leq b\left\|\left\{g_{i}\right\}_{i \in \mathbb{J}}\right\|^{2}, \quad \forall\left\{g_{i}\right\}_{i \in \mathbb{J}} \in \bigoplus_{i \in \mathbb{J}} H_{i},
$$

we call $\left\{A_{i}\right\}_{i \in \mathbb{J}}$ a $g$-Riesz basis for $H$. As we know, if $\left\{A_{i}\right\}_{i \in \mathbb{J}}$ is a g-frame for $H$, we define $S_{A} f=\sum_{i \in \mathbb{J}} A_{i}^{*} A_{i} f$ for any $f \in H$, then $S_{A}$ is a well-defined, bounded, positive, invertible operator by [12]. We call $S_{A}$ a frame operator of $\left\{A_{i}\right\}_{i \in \mathbb{J}}$. Another basic fact is that $\left\{\tilde{A}_{i}\right.$ : $\left.\widetilde{A}_{i}=A_{i} S_{A}^{-1}\right\}_{i \in \mathbb{J}}$ is a g-frame for $H$, we call it a canonical dual g-frame of $\left\{A_{i}\right\}_{i \in \mathbb{J}}$. Extensively, by [10], if $\left\{B_{i}\right\}_{i \in \mathbb{J}}$ is a $\mathrm{g}$-frame for $H$ such that $f=\sum_{i \in \mathbb{J}} B_{i}^{*} A_{i} f$ for every $f \in H$, we say that 
it is a dual $g$-frame of $\left\{A_{i}\right\}_{i \in \mathbb{J}}$. Actually, g-frames and operator valued frames (see $\left.[9,10]\right)$ are equivalent. Recently, g-frames or operator valued frames in Hilbert spaces have been studied intensively; for more details, see $[1,2,7,8,10-12]$ and the references therein.

In order to understand the structured g-frames more deeply, Guo studied the wandering generators for a unitary system in [6]. In [10] and [9], the authors studied the frame generators for group representations. In [11] the author studied the frame generator for a group-like unitary system. These researchers showed that such an abstract way to study the structured g-frames is very feasible and fruitful. We denote a unitary representation $\pi$ for a countable group $\mathcal{G}$ by $(\mathcal{G}, \pi, H)$, which is a mapping $g \mapsto \pi(g)$ from $\mathcal{G}$ into the set of unitary operators on a Hilbert space $H$ such that $\pi(g) \pi(h)=\pi(g h)$ for any $g, h \in \mathcal{G}$. In this paper, we focus on the dilation problem on frame generator dual pairs for a unitary representation in Hilbert spaces. Firstly, in order to establish our techniques, we give a direct proof for the existence of a Riesz generator dilation dual pair of a frame generator dual pair in Hilbert spaces. As there may be more than one dual frame generator of a given frame generator in general, there may be different pairs of frame generator dual pairs. Our main result characterizes the relations between the dilations of all these pairs. We illustrate that all the dilations can be mutually transformed by a type of special structured lower triangular operator matrices.

Throughout this paper, $H, H_{0}$ denote separable Hilbert spaces. Let $B\left(H, H_{0}\right)$ denote all the bounded linear operators from $H$ to $H_{0}$ and $B(H):=B(H, H)$. If $M, N$ are closed subspaces of $H, H=M+N$ denotes that $M+N=H$ and $M \cap N=\{0\}$. We use $M^{\perp}$ to denote the orthogonal complement of a closed subspace $M$ contained in $H$. For an operator $T \in B\left(H, H_{0}\right)$, we let $\operatorname{ker} T$ denote the null space of $T$ and $\operatorname{ran} T$ denote the range space of $T$. For a subset $\mathcal{S} \subset B(H), \mathcal{S}^{\prime}$ denotes the commutant of $\mathcal{S}$. We denote by $A \pi(\mathcal{G}):=\{A \pi(g)\}_{g \in \mathcal{G}}$ for $A \in B\left(H, H_{0}\right)$.

Definition 1.1 ([10]) Let $(\mathcal{G}, \pi, H)$ be a unitary representation of the countable group $\mathcal{G}$ on a Hilbert space $H$. Suppose $A \in B\left(H, H_{0}\right)$. Then

(1) $A$ is called a Bessel generator of $(\mathcal{G}, \pi, H)$ if $A \pi(\mathcal{G})$ is a g-Bessel sequence for $H$.

(2) $A$ is called $a$ (resp. Parseval, tight) frame generator of $(\mathcal{G}, \pi, H)$ if $A \pi(\mathcal{G})$ is a (resp. Parseval, tight) g-frame for $H$.

(3) $A$ is called $a$ Riesz (resp. orthonormal) generator of $(\mathcal{G}, \pi, H)$ if $A \pi(\mathcal{G})$ is a g-Riesz (resp. g-orthonormal) basis for $H$.

Let $A \in B\left(H, H_{0}\right)$ be a Bessel generator of $(\mathcal{G}, \pi, H)$. For any $f \in H$, the analysis operator of $A$ is defined as

$$
\theta_{A}: H \rightarrow l^{2}(\mathcal{G}) \otimes H_{0}, \quad \theta_{A} f=\sum_{g \in \mathcal{G}} \chi_{g} \otimes A \pi(g)^{*} f
$$

where $\left\{\chi_{g}\right\}_{g \in \mathcal{G}}$ is the orthonormal basis for $l^{2}(\mathcal{G})$. And the frame operator of $A$ is defined as

$$
S_{A}: H \rightarrow H, \quad S_{A} f=\sum_{g \in \mathcal{G}} \pi(g) A^{*} A \pi(g)^{*} f .
$$


By [10], for any $k \in H_{0}$, the left regular representation of $\mathcal{G}$ on $l^{2}(\mathcal{G}) \otimes H_{0}$ is defined as

$$
\Lambda(g)\left(\chi_{e} \otimes k\right)=\left(\lambda_{g} \otimes I_{H_{0}}\right)\left(\chi_{e} \otimes k\right)=\chi_{g} \otimes k, \quad \forall g \in \mathcal{G}, k \in H_{0},
$$

where $\lambda$ denotes the left regular representation of $\mathcal{G}$ on $l^{2}(\mathcal{G}), I_{H_{0}}$ is the identity operator on $H_{0}, e \in \mathcal{G}$ is the unit element.

For every $g \in \mathcal{G}$, define $Q_{g}: l^{2}(\mathcal{G}) \otimes H_{0} \rightarrow H_{0}$ as

$$
Q_{g}\left(\chi_{h} \otimes k\right)=\delta_{g, h} k, \quad \forall h \in \mathcal{G}, k \in H_{0}
$$

where $\delta_{g, h}=1$ for $h=g$ and $\delta_{g, h}=0$ for $h \neq g$.

Lemma $1.2([11]) Q_{h}$ is an orthonormal generator of $\left(\mathcal{G}, \Lambda, l^{2}(\mathcal{G}) \otimes H_{0}\right)$ for any $h \in \mathcal{G}$.

Definition 1.3 Let $(\mathcal{G}, \pi, H)$ be a unitary representation of $\mathcal{G}$ on $H$. If $A, B \in B\left(H, H_{0}\right)$ such that $B \pi(\mathcal{G})$ is a (canonical) dual g-frame (resp. g-Riesz basis) of the g-frame (resp. g-Riesz basis) $A \pi(\mathcal{G})$, we call $B$ a (canonical) dual frame (resp. Riesz) generator of $A$ (with the same structure). In this case, $(A, B)$ is called a pair of dual frame (resp. Riesz) generators of $(\mathcal{G}, \pi, H)$ or a frame (resp. Riesz) generator dual pair.

Lemma 1.4 Let $(\mathcal{G}, \pi, H)$ be a unitary representation of $\mathcal{G}$ on $H, A, B$ be any Bessel generators of $(\mathcal{G}, \pi, H)$. Then $\theta_{A} \theta_{B}^{*} \in \Lambda(\mathcal{G})^{\prime}$.

Proof For any $g, h \in \mathcal{G}, k \in H_{0}$, we have

$$
\begin{aligned}
\theta_{A} \theta_{B}^{*}\left(\Lambda(g)\left(\chi_{h} \otimes k\right)\right) & =\theta_{A} \pi(g h) B^{*} k=\sum_{g^{\prime} \in \mathcal{G}} \chi_{g^{\prime}} \otimes A \pi\left(g^{\prime}\right)^{*} \pi(g h) B^{*} k \\
& =\sum_{g^{\prime} \in \mathcal{G}} \Lambda(g)\left(\chi_{g^{\prime}} \otimes A \pi\left(g^{\prime}\right)^{*} \pi(h) B^{*} k\right) \\
& =\Lambda(g) \theta_{A} \theta_{B}^{*}\left(\chi_{h} \otimes k\right) .
\end{aligned}
$$

Theorem 1.5 Let $(\mathcal{G}, \pi, H)$ be a unitary representation of $\mathcal{G}$ on $H$. Then the following are equivalent:

(1) $(A, B)$ is a frame generator dual pair of $(\mathcal{G}, \pi, H)$, where $A, B \in B\left(H, H_{0}\right)$.

(2) There exist a Hilbert space $K \supset H$, a unitary representation $(\mathcal{G}, \sigma, K)$ of $\mathcal{G}$ on $K$, and a Riesz generator dual pair $(C, D)$ of $(\mathcal{G}, \sigma, K)$ such that $C P=A, D P=B, H$ is $\sigma$-invariant and $\pi=\left.\sigma\right|_{H}$, where $C, D \in B\left(K, H_{0}\right), P$ is the orthogonal projection from $K$ onto $H$.

Proof Suppose that $(A, B)$ is a frame generator dual pair of $(\mathcal{G}, \pi, H)$. Let $M=\operatorname{ran} \theta_{A}, N=$ $\operatorname{ran} \theta_{B}, P_{A}, P_{B}$ be the orthogonal projection from $l^{2}(\mathcal{G}) \otimes H_{0}$ onto $M, N$ respectively. We can easily get that

$$
P_{A}=\theta_{A S_{A}^{-\frac{1}{2}}} \theta_{A S_{A}^{-\frac{1}{2}}}^{*}=\theta_{A} S_{A}^{-1} \theta_{A}^{*}
$$


And then $P_{A} \theta_{B}=\theta_{A} S_{A}^{-1}$. It follows that $\left.P_{A}\right|_{N}: N \rightarrow M$ is invertible. Let $I_{l^{2}}$ be the identity operator on $l^{2}(\mathcal{G}) \otimes H_{0}$. Since

$$
I_{l^{2}}=\left(\begin{array}{cc}
P_{A} & 0 \\
0 & P_{A}^{\perp}
\end{array}\right): \quad\left(\begin{array}{c}
N \\
N^{\perp}
\end{array}\right) \rightarrow\left(\begin{array}{c}
M \\
M^{\perp}
\end{array}\right),
$$

$P_{A}^{\perp}: N^{\perp} \rightarrow M^{\perp}$ is invertible. Similarly, $P_{B}^{\perp}: M^{\perp} \rightarrow N^{\perp}$ is invertible.

Let $K=H \oplus N^{\perp}, \sigma(g)=\pi(g) \oplus \Lambda(g)$ for any $g \in \mathcal{G}, C=A \oplus Q_{e} P_{B}^{\perp} \in B\left(K, H_{0}\right)$. Thus $\sigma(g) C^{*}=\pi(g) A^{*} \oplus \Lambda(g) P_{B}^{\perp} Q_{e}^{*}$ for any $g \in \mathcal{G}$. Hence, by Lemma 1.4, we have

$$
\sigma(g) C^{*}=\pi(g) A^{*} \oplus P_{B}^{\perp} \Lambda(g) Q_{e}^{*}
$$

Therefore, for every $x \in H, y \in N^{\perp}$, we get

$$
\begin{aligned}
\theta_{C}(x \oplus y) & =\sum_{g \in \mathcal{G}} \chi_{g} \otimes C \sigma(g)^{*}(x \oplus y) \\
& =\sum_{g \in \mathcal{G}} \chi_{g} \otimes\left(A \pi(g)^{*} x+Q_{e} \Lambda(g)^{*} P_{B}^{\perp} y\right) \\
& =\theta_{A} x+P_{B}^{\perp} y .
\end{aligned}
$$

Let $T=\theta_{A} \theta_{B}^{*}$. Then $T^{2}=T$. We get $l^{2}(\mathcal{G}) \otimes H_{0}=M \dot{+} N^{\perp}$. It follows that $\theta_{C}$ is invertible. Hence $C$ is a Riesz generator of $(\mathcal{G}, \sigma, K)$. Obviously, $A=C P$, where $P$ is the orthogonal projection from $K$ onto $H$, and $H$ is $\sigma$-invariant.

Since $\rho:=P_{A}^{\perp} P_{B}^{\perp}: N^{\perp} \rightarrow M^{\perp}$ is invertible, there exists $\tau \in B\left(M^{\perp}, N^{\perp}\right)$ such that $\tau \rho=P_{B}^{\perp}$. Let $D=B \oplus Q_{e} P_{A}^{\perp} \tau^{*} \in B\left(K, H_{0}\right)$.

Let $\Lambda_{1}(g)=P_{A}^{\perp} \Lambda(g) P_{A}^{\perp}, \Lambda_{2}(g)=P_{B}^{\perp} \Lambda(g) P_{B}^{\perp}$ for any $g \in \mathcal{G}$. Then, for arbitrary $u \in N^{\perp}$,

$$
\begin{aligned}
\rho \Lambda_{2}(g) u & =\rho P_{B}^{\perp} \Lambda(g) P_{B}^{\perp} u=P_{A}^{\perp} P_{B}^{\perp} P_{B}^{\perp} \Lambda(g) P_{B}^{\perp} u \\
& =\Lambda(g) P_{A}^{\perp} P_{B}^{\perp} u=P_{A}^{\perp} \Lambda(g) P_{A}^{\perp} \rho u \\
& =\Lambda_{1}(g) \rho u .
\end{aligned}
$$

Hence, $\tau \Lambda_{1}(g) v=\Lambda_{2}(g) \tau v$ for every $v \in M^{\perp}$.

Therefore, for any $g \in \mathcal{G}$,

$$
\sigma(g) D^{*}=\pi(g) B^{*} \oplus \Lambda(g) \tau P_{A}^{\perp} Q_{e}^{*}=\pi(g) B^{*} \oplus \tau P_{A}^{\perp} \Lambda(g) Q_{e}^{*} .
$$

Obviously, $Q_{e} \Lambda(\mathcal{G}) P_{A}^{\perp} \tau^{*}=\left\{Q_{e} \Lambda(g) P_{A}^{\perp} \tau^{*}\right\}_{g \in \mathcal{G}}$ is a g-frame for $N^{\perp}$. It follows that $Q_{e} P_{A}^{\perp} \tau^{*}$ is a frame generator of $\left(\mathcal{G}, \Lambda, N^{\perp}\right)$.

Hence, for arbitrary $x \in H, y \in N^{\perp}$, we have

$$
\begin{aligned}
\theta_{D}(x \oplus y) & =\sum_{g \in \mathcal{G}} \chi_{g} \otimes D \sigma(g)^{*}(x \oplus y) \\
& =\sum_{g \in \mathcal{G}} \chi_{g} \otimes\left(B \pi(g)^{*} x+Q_{e} P_{A}^{\perp} \tau^{*} \Lambda(g)^{*} y\right) \\
& =\theta_{B} x+P_{A}^{\perp} \tau^{*} y .
\end{aligned}
$$


Similarly, since $l^{2}(\mathcal{G}) \otimes H_{0}=M^{\perp} \dot{+} N, \theta_{D}$ is invertible. Then $D$ is a Riesz generator of $(\mathcal{G}, \sigma, K)$ and $B=D P$.

For any $x, x_{1} \in H, y, y_{1} \in N^{\perp}$,

$$
\begin{aligned}
& \sum_{g \in \mathcal{G}}\left\langle\sigma(g) C^{*} D \sigma(g)^{*} x \oplus y, x_{1} \oplus y_{1}\right\rangle \\
&=\sum_{g \in \mathcal{G}}\left\langle\left(\pi(g) A^{*} \oplus P_{B}^{\perp} \Lambda(g) Q_{e}^{*}\right)\left(B \pi(g)^{*} \oplus Q_{e} \Lambda(g)^{*} P_{A}^{\perp} \tau^{*}\right)(x \oplus y), x_{1} \oplus y_{1}\right\rangle \\
&=\sum_{g \in \mathcal{G}}\left\langle\left(B \pi(g)^{*} \oplus Q_{e} \Lambda(g)^{*} P_{A}^{\perp} \tau^{*}\right)(x \oplus y),\left(A \pi(g)^{*} \oplus Q_{e} \Lambda(g)^{*} P_{B}^{\perp}\right)\left(x_{1} \oplus y_{1}\right)\right\rangle \\
&=\sum_{g \in \mathcal{G}}\left\langle B \pi(g)^{*} x+Q_{e} \Lambda(g)^{*} P_{A}^{\perp} \tau^{*} y, A \pi(g)^{*} x_{1}+Q_{e} \Lambda(g)^{*} P_{B}^{\perp} y_{1}\right\rangle \\
&=\sum_{g \in \mathcal{G}}\left\langle B \pi(g)^{*} x, A \pi(g)^{*} x_{1}\right\rangle+\sum_{g \in \mathcal{G}}\left\langle B \pi(g)^{*} x, Q_{e} \Lambda(g)^{*} P_{B}^{\perp} y_{1}\right\rangle \\
& \quad+\sum_{g \in \mathcal{G}}\left\langle Q_{e} \Lambda_{g}^{*} P_{A}^{\perp} \tau^{*} y, A \pi(g)^{*} x_{1}\right\rangle+\sum_{g \in \mathcal{G}}\left\langle Q_{e} \Lambda(g)^{*} P_{A}^{\perp} \tau^{*} y, Q_{e} \Lambda(g)^{*} P_{B}^{\perp} y_{1}\right\rangle . \\
&=\left\langle\theta_{B} x, \theta_{A} x_{1}\right\rangle+\left\langle\theta_{B} x, P_{B}^{\perp} y_{1}\right\rangle+\left\langle P_{A}^{\perp} \tau^{*} y, \theta_{A} x_{1}\right\rangle+\left\langle P_{A}^{\perp} \tau^{*} y, P_{B}^{\perp} y_{1}\right\rangle \\
&=\left\langle x, x_{1}\right\rangle+\left\langle y, y_{1}\right\rangle=\left\langle x \oplus y, x_{1} \oplus y_{1}\right\rangle,
\end{aligned}
$$

which implies $(C, D)$ is a Riesz generator dual pair of $(\sigma, \mathcal{G}, K)$.

The converse is obvious.

Remark 1.6 (1) From the proof above, the isomorphism of $N, M$ is critical. In the following we provide an easier way of the proof.

In fact, as above, $C \in B\left(K, H_{0}\right)$ is a Riesz generator of $(\mathcal{G}, \sigma, K)$. So $S_{C} \in \sigma(\mathcal{G})^{\prime}$. And then, $C S_{C}^{-1} \in B\left(K, H_{0}\right)$ is a Riesz generator of $(\mathcal{G}, \sigma, K)$ evidently.

Let $C S_{C}^{-1}=D_{1} \oplus D_{2}$, where $D_{1} \in B\left(H, H_{0}\right), D_{2} \in B\left(N^{\perp}, H_{0}\right)$. Then $D_{1}=C S_{C}^{-1} P$. We need to show $D_{1}=B$.

Identify $H$ with $H \oplus\{0\}$. For arbitrary $x, x_{1} \in H$,

$$
\begin{aligned}
\left\langle x, x_{1}\right\rangle & =\left\langle x \oplus 0, x_{1} \oplus 0\right\rangle \\
& =\sum_{g \in \mathcal{G}}\left\langle\sigma(g) C^{*} C S_{C}^{-1} \sigma(g)^{*} x \oplus 0, x_{1} \oplus 0\right\rangle \\
& =\sum_{g \in \mathcal{G}}\left\langle\left(\pi(g) A^{*} \oplus P_{B}^{\perp} \Lambda(g) Q_{e}^{*}\right)\left(D_{1} \pi(g)^{*} \oplus D_{2} \Lambda(g)^{*}\right)(x \oplus 0), x_{1} \oplus 0\right\rangle \\
& =\sum_{g \in \mathcal{G}}\left\langle\left(D_{1} \pi(g)^{*} \oplus D_{2} \Lambda(g)^{*}\right)(x \oplus 0),\left(A \pi(g)^{*} \oplus Q_{e} \Lambda(g)^{*} P_{B}^{\perp}\right)\left(x_{1} \oplus 0\right)\right\rangle \\
& =\sum_{g \in \mathcal{G}}\left\langle D_{1} \pi(g)^{*} x, A \pi(g)^{*} x_{1}\right\rangle,
\end{aligned}
$$

which means $D_{1} \in B\left(H, H_{0}\right)$ is a dual frame generator of $A$.

Moreover, for any $x, x_{1} \in H, y_{1} \in N^{\perp}$.

$$
\left\langle x, x_{1}\right\rangle=\left\langle x \oplus 0, x_{1} \oplus y_{1}\right\rangle
$$




$$
\begin{aligned}
& =\sum_{g \in \mathcal{G}}\left\langle\sigma(g) C^{*} C S_{C}^{-1} \sigma(g)^{*} x \oplus 0, x_{1} \oplus y_{1}\right\rangle \\
& =\sum_{g \in \mathcal{G}}\left\langle D_{1} \pi(g)^{*} x, A \pi(g)^{*} x_{1}+Q_{e} P_{B}^{\perp} \Lambda(g)^{*} y_{1}\right\rangle \\
& =\left\langle x, x_{1}\right\rangle+\sum_{g \in \mathcal{G}}\left\langle D_{1} \pi(g)^{*} x, Q_{e} P_{B}^{\perp} \Lambda(g)^{*} y_{1}\right\rangle .
\end{aligned}
$$

Then

$$
\sum_{g \in \mathcal{G}}\left\langle D_{1} \pi(g)^{*} x, Q_{e} P_{B}^{\perp} \Lambda(g)^{*} y_{1}\right\rangle=\left\langle\theta_{D_{1}} x, y_{1}\right\rangle=0 .
$$

We get $\operatorname{ran} \theta_{D_{1}} \subseteq N=\operatorname{ran} \theta_{B}$. Hence, for every $x \in H$, there exists $x_{1} \in H$ such that $\theta_{D_{1}} x=$ $\theta_{B} x_{1}$. Then

$$
x=\theta_{A}^{*} \theta_{D_{1}} x=\theta_{A}^{*} \theta_{B} x_{1}=x_{1} .
$$

Therefore, for any $x \in H$, we obtain $\theta_{D_{1}} x=\theta_{B} x$, which implies $B=D_{1}$.

(2) We can also have another way to prove $B=D_{1}$ as follows.

For any $x, x_{1} \in H, y_{1} \in N^{\perp}$, on the one hand,

$$
\begin{aligned}
\left\langle x, x_{1}\right\rangle & =\left\langle x \oplus 0, x_{1} \oplus y_{1}\right\rangle \\
& =\sum_{g \in \mathcal{G}}\left\langle\sigma(g) C^{*} C S_{C}^{-1} \sigma(g)^{*} x \oplus 0, x_{1} \oplus y_{1}\right\rangle \\
& =\sum_{g \in \mathcal{G}}\left\langle D_{1} \pi(g)^{*} x, C \sigma(g)^{*}\left(x_{1} \oplus y_{1}\right)\right\rangle .
\end{aligned}
$$

On the other hand,

$$
\begin{aligned}
\sum_{g \in \mathcal{G}} & \left\langle B \pi(g)^{*} x, C \sigma(g)^{*}\left(x_{1} \oplus y_{1}\right)\right\rangle \\
& =\sum_{g \in \mathcal{G}}\left\langle B \pi(g)^{*} x, A \pi(g)^{*} x_{1}+Q_{1} P_{B}^{\perp} \Lambda(g)^{*} y_{1}\right\rangle \\
& =\left\langle x, x_{1}\right\rangle .
\end{aligned}
$$

Hence, $\theta_{C}^{*} \theta_{B} x=\theta_{C}^{*} \theta_{D_{1}} x$. Since $\theta_{C}$ is invertible, it follows that $\theta_{B} x=\theta_{D_{1}} x$, which means $B=$ $D_{1}$.

Definition 1.7 Let $(\mathcal{G}, \pi, H),\left(\mathcal{G}, \pi_{1}, N_{1}\right)$ be unitary representations of $\mathcal{G}$ on $H, N_{1}$ respectively, $(A, B)$ be a pair of dual frame generators of $(\mathcal{G}, \pi, H)$, where $A, B \in B\left(H, H_{0}\right)$. If there exist $C_{1}, D_{1} \in B\left(N_{1}, H_{0}\right)$ such that $(E, \widetilde{E})$ is a Riesz generator dual pair of $\left(\mathcal{G}, \sigma_{1}, H \oplus N_{1}\right)$, where $\sigma_{1}=\pi \oplus \pi_{1}, E=A \oplus C_{1}, \widetilde{E}=B \oplus D_{1}$. We call $(E, \widetilde{E})$ a dilation of $(A, B) .\left(C_{1}, D_{1}\right)$ is called a complementary generator pair of $(A, B)$. Particularly, $C_{1}$ is called a complementary generator of $A$.

Theorem 1.5 illustrates that there exists a dilation for any pair of dual frame generators. 
In the next we show that the dilation of a dual frame generator pair is unique in the sense of "similarity".

Theorem 1.8 Let $(\mathcal{G}, \pi, H),\left(\mathcal{G}, \pi_{1}, N_{1}\right),\left(\mathcal{G}, \pi_{2}, N_{2}\right)$ be unitary representations of $\mathcal{G}$ on $H, N_{1}$, $N_{2}$ respectively, $(A, B)$ be a pair of dual frame generators of $(\mathcal{G}, \pi, H)$, where $A, B \in B\left(H, H_{0}\right)$. Suppose that $(E, \widetilde{E})$ is a Riesz generator dual pair of $\left(\mathcal{G}, \sigma_{1}, H \oplus N_{1}\right)$ which is also a dilation of $(A, B)$, where $\sigma_{1}=\pi \oplus \pi_{1}, E=A \oplus C_{1}, C_{1} \in B\left(N_{1}, H_{0}\right), \widetilde{E}$ is the canonical dual Riesz generator of $E$. If there exist $C_{2} \in B\left(N_{2}, H_{0}\right)$ and an invertible operator $T \in B\left(N_{1}, N_{2}\right)$ such that $C_{1} \pi_{1}(g)=C_{2} \pi_{2}(g) T$ for every $g \in \mathcal{G}$, then $F$ is a Riesz generator of $\left(\mathcal{G}, \sigma_{2}, H \oplus N_{2}\right)$, where $\sigma_{2}=\pi \oplus \pi_{2}, F=A \oplus C_{2} \in B\left(H \oplus N_{2}, H_{0}\right)$. Moreover, $(F, \widetilde{F})$ is a Riesz generator dual pair of $\left(\mathcal{G}, \sigma_{2}, H \oplus N_{2}\right)$ which is also a dilation of $(A, B)$, where $\widetilde{F}$ is the canonical dual Riesz generator of $F$.

Proof Since $C_{1} \pi_{1}(g)=C_{2} \pi_{2}(g) T$ for any $g \in \mathcal{G}$, we have

$$
F \sigma_{2}(g)=A \pi(g) \oplus C_{2} \pi_{2}(g)=A \pi(g) \oplus C_{1} \pi_{1}(g) T^{-1}=E \sigma_{1}(g)\left(I \oplus T^{-1}\right),
$$

which implies $F$ is a Riesz generator of $\left(\mathcal{G}, \sigma_{2}, H \oplus N_{2}\right)$ and $A=F P_{2}$, where $P_{2}$ is the orthogonal projection from $H \oplus N_{2}$ onto $H$.

Denote $\widehat{T}=I \oplus T$. As $\widetilde{F}=F S_{F}^{-1}$, it follows that, for every $g \in \mathcal{G}$,

$$
\widetilde{F} \sigma_{2}(g)=F \sigma_{2}(g) S_{F}^{-1}=E \sigma_{1}(g) \widehat{T}^{-1} S_{F}^{-1}=\widetilde{E} \sigma_{1}(g) S_{E} \widehat{T}^{-1} S_{F}^{-1} .
$$

Besides, we can get $S_{F}=\theta_{F}^{*} \theta_{F}=\left(\widehat{T}^{*}\right)^{-1} S_{E} \widehat{T}^{-1}$. Then

$$
\widetilde{F} \sigma_{2}(g)=\widetilde{E} \sigma_{1}(g) \widehat{T}^{*}=\widetilde{E} \sigma_{1}(g)\left(I \oplus T^{*}\right) .
$$

Evidently, $\widetilde{F} \sigma_{2}(g) P_{2}=B \pi(g)$. Specially, we have $\widetilde{F} P_{2}=B$.

In the following we exhibit that two different complementary generators of a given frame generator are "similar".

Theorem 1.9 Let $(\mathcal{G}, \pi, H),\left(\mathcal{G}, \pi_{1}, N_{1}\right),\left(\mathcal{G}, \pi_{2}, N_{2}\right)$ be unitary representations of $\mathcal{G}$ on $H, N_{1}$, $N_{2}$ respectively, $(A, B)$ be a pair of dual frame generators of $(\mathcal{G}, \pi, H)$, where $A, B \in B\left(H, H_{0}\right)$. Suppose that $(E, \widetilde{E})$ is a Riesz generator dual pair of $\left(\mathcal{G}, \sigma_{1}, H \oplus N_{1}\right)$ which is also a dilation of $(A, B)$, where $\sigma_{1}=\pi \oplus \pi_{1}, E=A \oplus C_{1}, C_{1} \in B\left(N_{1}, H_{0}\right), \widetilde{E}$ is the canonical dual Riesz generator of $E$. If $(F, \widetilde{F})$ is a Riesz generator dual pair of $\left(\mathcal{G}, \sigma_{2}, H \oplus N_{2}\right)$ which is also a dilation of $(A, B)$, where $\sigma_{2}=\pi \oplus \pi_{2}, F=A \oplus C_{2}, C_{2} \in B\left(N_{2}, H_{0}\right), \widetilde{F}$ is the canonical dual Riesz generator of $F$, then there exists an invertible operator $T \in B\left(N_{1}, N_{2}\right)$ such that $C_{1} \pi_{1}(g)=C_{2} \pi_{2}(g) T$ for every $g \in \mathcal{G}$. In particular, $T \pi_{1}(g)=\pi_{2}(g) T$.

Proof Let $\widetilde{E}=B \oplus D_{1}, \widetilde{F}=B \oplus D_{2}$, where $D_{1} \in B\left(N_{1}, H_{0}\right), D_{2} \in B\left(N_{2}, H_{0}\right)$. Then, for every $x, y \in H, x_{1} \in N_{1}$, we get

$$
\begin{aligned}
\langle x, y\rangle & =\left\langle x \oplus 0, y \oplus x_{1}\right\rangle=\left\langle\theta_{E}^{*} \theta_{\widetilde{E}}(x \oplus 0), y \oplus x_{1}\right\rangle \\
& =\sum_{g \in \mathcal{G}}\left\langle B \pi(g)^{*} x, A \pi(g)^{*} y+C_{1} \pi_{1}(g)^{*} x_{1}\right\rangle \\
& =\langle x, y\rangle+\left\langle x, \theta_{B}^{*} \theta_{C_{1}} x_{1}\right\rangle .
\end{aligned}
$$


Thus $\theta_{B}^{*} \theta_{C_{1}}=0$, which implies $\operatorname{ran} \theta_{C_{1}} \subseteq\left(\operatorname{ran} \theta_{B}\right)^{\perp}$. Since $E$ is a Riesz generator of $\left(\mathcal{G}, \sigma_{1}, H \oplus\right.$ $N_{1}$ ), by [2, Proposition 2.3] we have

$$
l^{2}(\mathcal{G}) \otimes H_{0}=\operatorname{ran} \theta_{A} \dot{+} \operatorname{ran} \theta_{C_{1}} .
$$

Hence, for any $u \in\left(\operatorname{ran} \theta_{B}\right)^{\perp}$, there exist $x \in H, x_{1} \in N_{1}$ such that $u=\theta_{A} x+\theta_{C_{1}} x_{1}$. Then, $0=\theta_{B}^{*} u=\theta_{B}^{*} \theta_{A} x+\theta_{B}^{*} \theta_{C_{1}} x_{1}=x$, therefore, $u=\theta_{C_{1}} x_{1} \in \operatorname{ran} \theta_{C_{1}}$, equivalently, $\left(\operatorname{ran} \theta_{B}\right)^{\perp} \subseteq$ $\operatorname{ran} \theta_{C_{1}}$. Therefore, we obtain $\operatorname{ran} \theta_{C_{1}}=\left(\operatorname{ran} \theta_{B}\right)^{\perp}$.

Similarly, we obtain $\operatorname{ran} \theta_{C_{2}}=\left(\operatorname{ran} \theta_{B}\right)^{\perp}$. Then $\operatorname{ran} \theta_{C_{1}}=\operatorname{ran} \theta_{C_{2}}$. Hence, by [10, Proposition 4.3], there is an invertible operator $T \in B\left(N_{1}, N_{2}\right)$ such that $C_{1} \pi_{1}(g)=C_{2} \pi_{2}(g) T$ for arbitrary $g \in \mathcal{G}$.

Specially, for every $x_{1} \in N_{1}$, we have $x=\sum_{g \in \mathcal{G}} \pi_{1}(g) C_{1}^{*} \widetilde{C}_{1} \pi_{1}(g)^{*} x$, where $\widetilde{C}_{1} \in B\left(N_{1}, H_{0}\right)$ is the canonical dual frame generator of $C_{1}$. Besides, for any $h \in \mathcal{G}$,

$$
\begin{aligned}
\pi_{2}(h)\left(T^{*}\right)^{-1} x & =\sum_{g \in \mathcal{G}} \pi_{2}(h)\left(T^{*}\right)^{-1} \pi_{1}(g) C_{1}^{*} \widetilde{C}_{1} \pi_{1}(g)^{*} x \\
& =\sum_{g \in \mathcal{G}} \pi_{2}(h g) C_{2}^{*} \widetilde{C}_{1} \pi_{1}(h g)^{*} \pi_{1}(h) x \\
& =\sum_{g \in \mathcal{G}}\left(T^{*}\right)^{-1} \pi_{1}(h g) C_{1}^{*} \widetilde{C}_{1} \pi_{1}(h g)^{*} \pi_{1}(h) x \\
& =\left(T^{*}\right)^{-1} \pi_{1}(h) x,
\end{aligned}
$$

which implies $T \pi_{1}(g)=\pi_{2}(g) T$ for every $g \in \mathcal{G}$.

By the proof of Theorem 1.8 and Theorem 1.9, two complementary generator pairs of a frame generator dual pair are "similar" by the operator pair $\left(T, T^{*}\right)$, which implies that complementary generator pairs are unique in the sense of "similarity".

\section{Characterization of all the dilations of a frame generator dual pair}

The following is a result which describes the dilation of the frame generator canonical dual pair.

Theorem 2.1 Let $(\mathcal{G}, \pi, H)$ be a unitary representation of $\mathcal{G}$ on $H,(A, \widetilde{A})$ be a pair of dual frame generators of $(\mathcal{G}, \pi, H)$, where $A \in B\left(H, H_{0}\right), \widetilde{A}$ is the canonical dual frame generator of $A$. Denote $E=A \oplus Q_{e} P_{A}^{\perp}, \widetilde{E}=\widetilde{A} \oplus Q_{e} P_{A}^{\perp} \in B\left(H \oplus M^{\perp}, H_{0}\right)$, where $M=\operatorname{ran} \theta_{A}, P_{A}$ is the orthogonal projection from $l^{2}(\mathcal{G}) \otimes H_{0}$ onto $M$. Then $(E, \widetilde{E})$ is a pair of dual Riesz generators of $\left(\mathcal{G}, \sigma, H \oplus M^{\perp}\right)$ which is a dilation of $(A, \widetilde{A})$, where $\sigma:=\pi \oplus \Lambda,\left(\mathcal{G}, \Lambda, l^{2}(\mathcal{G}) \otimes H_{0}\right)$ is the left regular representation of $\mathcal{G}$.

Proof Let $C=Q_{e} P_{A}^{\perp} \in B\left(M^{\perp}, H_{0}\right)$. By Lemma 1.4, $C \Lambda(\mathcal{G})$ is a Parseval g-frame for $M^{\perp}$ and

$$
\operatorname{ran} \theta_{A}=\operatorname{ran} \theta_{\widetilde{A}}=M, \quad \operatorname{ran} \theta_{C}=M^{\perp} .
$$

Since $\theta_{E}(x \oplus y)=\theta_{A} x+\theta_{C} y$ for any $x \in H, y \in M^{\perp}$, we have $\theta_{E}$ is invertible, which means $E$ is a Riesz generator of $\left(\mathcal{G}, \sigma, H \oplus M^{\perp}\right)$. Similarly, $\widetilde{E}$ is also a Riesz generator of $\left(\mathcal{G}, \sigma, H \oplus M^{\perp}\right)$. 
For arbitrary $x, y \in H, x_{1}, y_{1} \in M^{\perp}$,

$$
\begin{aligned}
\left\langle\sum_{g \in \mathcal{G}} \sigma(g) E^{*} \widetilde{E} \sigma(g)^{*}\left(x \oplus x_{1}\right), y \oplus y_{1}\right\rangle \\
=\left\langle\sum_{g \in \mathcal{G}}\left(\pi(g) A^{*} \oplus \Lambda(g) P_{A}^{\perp} Q_{e}^{*}\right)\left(\widetilde{A} \pi(g)^{*} \oplus Q_{e} P_{A}^{\perp} \Lambda(g)^{*}\right)\left(x \oplus x_{1}\right), y \oplus y_{1}\right\rangle \\
=\sum_{g \in \mathcal{G}}\left\langle\widetilde{A} \pi(g)^{*} x+Q_{e} P_{A}^{\perp} \Lambda(g)^{*} x_{1}, A \pi(g)^{*} y+Q_{e} P_{A}^{\perp} \Lambda(g)^{*} y_{1}\right\rangle \\
=\sum_{g \in \mathcal{G}}\left\langle\widetilde{A} \pi(g)^{*} x, A \pi(g)^{*} y\right\rangle+\sum_{g \in \mathcal{G}}\left\langle\widetilde{A} \pi(g)^{*} x, Q_{e} P_{A}^{\perp} \Lambda(g)^{*} y_{1}\right\rangle \\
\quad+\sum_{g \in \mathcal{G}}\left\langle Q_{e} P_{A}^{\perp} \Lambda(g)^{*} x_{1}, A \pi(g)^{*} y\right\rangle+\sum_{g \in \mathcal{G}}\left\langle Q_{e} P_{A}^{\perp} \Lambda(g)^{*} x_{1}, Q_{e} P_{A}^{\perp} \Lambda(g)^{*} y_{1}\right\rangle \\
=\sum_{g \in \mathcal{G}}\left\langle\widetilde{A} \pi(g)^{*} x, A \pi(g)^{*} y\right\rangle+\sum_{g \in \mathcal{G}}\left\langle Q_{e} P_{A}^{\perp} \Lambda(g)^{*} x_{1}, Q_{e} P_{A}^{\perp} \Lambda(g)^{*} y_{1}\right\rangle \\
=\langle x, y\rangle+\left\langle x_{1}, y_{1}\right\rangle=\left\langle x \oplus x_{1}, y \oplus y_{1}\right\rangle,
\end{aligned}
$$

which means $(E, \widetilde{E})$ is a pair of dual Riesz generators of $\left(\mathcal{G}, \sigma, H \oplus M^{\perp}\right)$.

We call the dilation $(E, \widetilde{E})$ in Theorem 2.1 the natural dilation of $(A, \widetilde{A})$. If $A \in B\left(H, H_{0}\right)$ is a frame generator of $(\mathcal{G}, \pi, H)$, let $M=\operatorname{ran} \theta_{A}, \sigma=\pi \oplus \Lambda, P_{A}$ be the orthogonal projection from $l^{2}(\mathcal{G}) \otimes H_{0}$ onto $M$. We can characterize all the dilations of frame generator dual pairs in terms of the natural dilation by a type of special lower triangular operator matrices.

Theorem 2.2 Let $(\mathcal{G}, \pi, H)$ be a unitary representation of $\mathcal{G}$ on $H,(A, B)$ be a frame generator alternate dual pair of $(\mathcal{G}, \pi, H)$, where $A, B \in B\left(H, H_{0}\right)$. Then there exists a pair of dual g-Riesz bases $\left(E^{\prime}, \widetilde{E}^{\prime}\right)$ which is a dilation of the g-frame dual pair $(A \pi(\mathcal{G}), B \pi(\mathcal{G}))$ such that $E^{\prime}:=\left\{E_{g}^{\prime}=E \sigma(g) T^{*}\right\}_{g \in \mathcal{G}}$, where $\widetilde{E}^{\prime}:=\left\{\widetilde{E}_{g}^{\prime}\right\}_{g \in \mathcal{G}}$ is the canonical dual g-frame of $E^{\prime}$,

$$
T=\left(\begin{array}{cc}
I_{H} & 0 \\
T^{\prime} & I_{M^{\perp}}
\end{array}\right) \in B\left(H \oplus M^{\perp}\right),
$$

$T^{\prime} \in B\left(H, M^{\perp}\right)$. (Note that $(E, \widetilde{E})$ is the natural dilation of $(A, \widetilde{A})$ in Theorem 2.1.)

Proof Let $\Gamma=\widetilde{A}-B \in B\left(H, H_{0}\right)$. Obviously, $\Gamma$ is a Bessel generator of $(\mathcal{G}, \pi, H)$. Then

$$
\theta_{\Gamma}^{*} \theta_{A}=-\theta_{B}^{*} \theta_{A}+\theta_{\widetilde{A}}^{*} \theta_{A}=0
$$

which implies $\operatorname{ran} \theta_{\Gamma} \perp \operatorname{ran} \theta_{A}$. So $T^{\prime}:=\theta_{\Gamma} \in B\left(H, M^{\perp}\right)$. Besides, $T=\left(\begin{array}{cc}I_{H} & 0 \\ T^{\prime} & I_{M^{\perp}}\end{array}\right) \in B\left(H \oplus M^{\perp}\right)$ is invertible. Hence, for any $g \in \mathcal{G}$,

$$
\begin{aligned}
E \sigma(g) T^{*} & =\left(A \pi(g) \oplus Q_{e} \Lambda(g) P_{A}^{\perp}\right)\left(\begin{array}{cc}
I_{H} & \left(T^{\prime}\right)^{*} \\
0 & I_{M^{\perp}}
\end{array}\right) \\
& =A \pi(g) \oplus\left(A \pi(g)\left(T^{\prime}\right)^{*}+Q_{e} \Lambda(g) P_{A}^{\perp}\right) .
\end{aligned}
$$


Evidently, $E^{\prime}:=\left\{E_{g}^{\prime}=E \sigma(g) T^{*}\right\}_{g \in \mathcal{G}}$ is a g-Riesz basis for $H \oplus M^{\perp}$ and $A \pi(g)=E \sigma(g) T^{*} P$ for every $g \in \mathcal{G}$, where $P$ is the orthogonal projection from $H \oplus M^{\perp}$ onto $H$. We can directly get $\widetilde{E}^{\prime}=\left\{\widetilde{E}_{g}^{\prime}:=\widetilde{E} \sigma(g) T^{-1}\right\}_{g \in \mathcal{G}}$ is the canonical dual g-Riesz basis of $E^{\prime}$. Moreover, for any $g \in \mathcal{G}$,

$$
\begin{aligned}
\widetilde{E} \sigma(g) T^{-1} & =\left(\widetilde{A} \pi(g) \oplus Q_{e} \Lambda(g) P_{A}^{\perp}\right)\left(\begin{array}{cc}
I_{H} & 0 \\
-T^{\prime} & I_{M^{\perp}}
\end{array}\right) \\
& =\left(\widetilde{A} \pi(g)-Q_{e} \Lambda(g) P_{A}^{\perp} T^{\prime}\right) \oplus Q_{e} \Lambda(g) P_{A}^{\perp} .
\end{aligned}
$$

Obviously, $\left(T^{\prime}\right)^{*} P_{A}^{\perp}=\left(T^{\prime}\right)^{*}$, where $P_{A}$ is the orthogonal projection from $l^{2}(\mathcal{G}) \otimes H_{0}$ onto $M$. And then, for every $g \in \mathcal{G}, k \in H_{0}$, we obtain

$$
\left(T^{\prime}\right)^{*} P_{A}^{\perp} \Lambda(g) Q_{e}^{*} k=-\pi(g) B^{*} k+\pi(g) \widetilde{A}^{*} k .
$$

Hence, $B \pi(g)=\widetilde{A} \pi(g)-Q_{e} \Lambda(g) P_{A}^{\perp} T^{\prime}$ which means $\widetilde{E}_{g}^{\prime}=B \pi(g) \oplus Q_{e} \Lambda(g) P_{A}^{\perp}$ and $B \pi(g)=$ $\widetilde{E}_{g}^{\prime} P$.

The next conclusion is more general than Theorem 2.2.

Theorem 2.3 Let $(\mathcal{G}, \pi, H),\left(\mathcal{G}, \pi_{2}, N\right)$ be unitary representations of $\mathcal{G}$ on $H, N$ respectively, $(A, B)$ be a frame generator alternate dual pair of $(\mathcal{G}, \pi, H)$, where $A, B \in B\left(H, H_{0}\right)$. If there is an invertible operator $\widetilde{T} \in B\left(M^{\perp}, N\right)$ such that $\widetilde{T} \Lambda(g)=\pi_{2}(g) \widetilde{T}$ for every $g \in \mathcal{G}$, then there exists a pair of dual Riesz generators $\left(E^{\prime}, \widetilde{E}^{\prime}\right)$ of $\left(\mathcal{G}, \sigma_{2}, H \oplus N\right)$ which is also a dilation of $(A, B)$ such that $E^{\prime} \sigma_{2}(g)=E \sigma(g) T^{*}$ for any $g \in \mathcal{G}$, where $\sigma_{2}=\pi \oplus \pi_{2}, \widetilde{E}^{\prime}$ is the canonical dual frame generator of $E^{\prime}, T=\left(\begin{array}{c}I_{H} \\ T^{\prime}\end{array}\right) \in B\left(H \oplus M^{\perp}, H \oplus N\right), T^{\prime} \in B(H, N)$. (Note that $(E, \widetilde{E})$ is the natural dilation of $(A, \widetilde{A})$ in Theorem 2.1.)

Proof Let $\Gamma=-B+\widetilde{A} \in B\left(H, H_{0}\right)$. It is easy to verify that $\Gamma$ is a Bessel generator of $(\mathcal{G}, \pi, H)$. Denote $T^{\prime}:=\widetilde{T} \theta_{\Gamma} \in B(H, N)$. Then $T$ is invertible and $T^{-1}=\left(\begin{array}{cc}I_{H} & 0 \\ -\widetilde{T}^{-1} T^{\prime} & \widetilde{T}^{-1}\end{array}\right)$.

Let $E_{g}^{\prime}=E \sigma(g) T^{*}$ for every $g \in \mathcal{G}$, thus

$$
\begin{aligned}
E_{g}^{\prime} & =E \sigma(g) T^{*}=\left(A \pi(g) \oplus Q_{e} \Lambda(g) P_{A}^{\perp}\right)\left(\begin{array}{cc}
I_{H} & \left(T^{\prime}\right)^{*} \\
0 & \widetilde{T}^{*}
\end{array}\right) \\
& =A \pi(g) \oplus\left(A \pi(g)\left(T^{\prime}\right)^{*}+Q_{e} \Lambda(g) P_{A}^{\perp} \widetilde{T}^{*}\right) \\
& =A \pi(g) \oplus\left(A \pi(g) \theta_{\Gamma}^{*} \widetilde{T}^{*}+Q_{e} \widetilde{T}^{*} \pi_{2}(g)\right) \\
& =A \pi(g) \oplus\left(\left(A \theta_{\Gamma}^{*} \widetilde{T}^{*}+Q_{e} \widetilde{T}^{*}\right) \pi_{2}(g)\right) \\
& =\left(A \oplus\left(A \theta_{\Gamma}^{*} \widetilde{T}^{*}+Q_{e} \widetilde{T}^{*}\right)\right)\left(\pi(g) \oplus \pi_{2}(g)\right) .
\end{aligned}
$$

Denote $E^{\prime}:=A \oplus\left(A \theta_{\Gamma}^{*} \widetilde{T}^{*}+Q_{e} \widetilde{T}^{*}\right) \in B\left(H \oplus N, H_{0}\right), \sigma_{2}=\pi \oplus \pi_{2}$. Therefore, $E^{\prime}$ is a Riesz generator of $\left(\mathcal{G}, \sigma_{2}, H \oplus N\right)$ and $A=E^{\prime} P$, where $P$ is the orthogonal projection from $H \oplus N$ onto $H$. Denote $\widetilde{E}_{g}^{\prime}:=\widetilde{E} \sigma(g) T^{-1}$ for any $g \in \mathcal{G}$. It is easy to examine $\left\{\widetilde{E}_{g}^{\prime}\right\}_{g \in \mathcal{G}}$ is the canonical dual g-frame of $\left\{E_{g}^{\prime}\right\}_{g \in \mathcal{G}}$. Then it is also a g-Riesz basis for $H \oplus N$. Moreover, for any $g \in \mathcal{G}$,

$$
\widetilde{E} \sigma(g) T^{-1}=\left(\widetilde{A} \pi(g) \oplus Q_{e} \Lambda(g) P_{A}^{\perp}\right) T^{-1}
$$




$$
\begin{aligned}
& =\left(\widetilde{A} \pi(g) \oplus Q_{e} \Lambda(g) P_{A}^{\perp}\right)\left(\begin{array}{cc}
I_{H} & 0 \\
-\widetilde{T}^{-1} T^{\prime} & \widetilde{T}^{-1}
\end{array}\right) \\
& =\left(\widetilde{A} \pi(g)-Q_{e} \Lambda(g) P_{A}^{\perp} \widetilde{T}^{-1} T^{\prime}\right) \oplus Q_{e} \Lambda(g) P_{A}^{\perp} \widetilde{T}^{-1} \\
& =\left(\widetilde{A} \pi(g)-Q_{e} \Lambda(g) P_{A}^{\perp} \theta_{\Gamma}\right) \oplus Q_{e} \Lambda(g) P_{A}^{\perp} \widetilde{T}^{-1} \\
& =(\widetilde{A} \pi(g)-(\widetilde{A} \pi(g)-B \pi(g))) \oplus Q_{e} \Lambda(g) P_{A}^{\perp} \widetilde{T}^{-1} \\
& =B \pi(g) \oplus Q_{e} \Lambda(g) \widetilde{T}^{-1} \\
& =B \pi(g) \oplus Q_{e} \widetilde{T}^{-1} \pi_{2}(g) .
\end{aligned}
$$

Denote $\widetilde{E}^{\prime}=B \oplus Q_{e} \widetilde{T}^{-1}$. Hence, $\widetilde{E}^{\prime}$ is a Riesz generator of $\left(\mathcal{G}, \sigma_{2}, H \oplus N\right)$ which is a dual frame generator of $E^{\prime}$ and such that $B=\widetilde{E}^{\prime} P$.

The following result illustrates that if a dilation g-Riesz basis pair of a frame generator alternate dual pair and that of the frame generator canonical dual pair are "similar" by a pair of operators, then one of the corresponding operators is in the form of $T$ in Theorem 2.3.

Theorem 2.4 Let $(\mathcal{G}, \pi, H)$ be a unitary representation of $\mathcal{G}$ on $H,(A, B)$ be a frame generator alternate dual pair of $(\mathcal{G}, \pi, H)$, where $A, B \in B\left(H, H_{0}\right)$.If there exists a pair of dual $g$ Riesz basis $\left(E^{\prime}, \widetilde{E}^{\prime}\right)$ for $H \oplus N$ which is also a dilation of the dual $g$-frame pair $(A \pi(\mathcal{G}), B \pi(\mathcal{G}))$ such that $E^{\prime}:=\left\{E_{g}^{\prime}=E \sigma(g) T^{*}\right\}_{g \in \mathcal{G}}$ for any $g \in \mathcal{G}$, where $N$ is a Hilbert space, $\widetilde{E}^{\prime}$ is the canonical dual $g$-Riesz basis of $E^{\prime}, T \in B\left(H \oplus M^{\perp}, H \oplus N\right)$ is invertible. Then $T$ has the following form:

$$
T=\left(\begin{array}{cc}
I_{H} & 0 \\
T^{\prime} & \widetilde{T}
\end{array}\right)
$$

where $T^{\prime} \in B(H, N), \widetilde{T} \in B\left(M^{\perp}, N\right)$. (Note that $(E, \widetilde{E})$ is the natural dilation of $(A, \widetilde{A})$ in Theorem 2.1.)

Proof As in the proofs of Theorem 2.2 and Theorem 2.3, let $\Gamma=-B+\widetilde{A} \in B\left(H, H_{0}\right)$. Then $\Gamma$ is a Bessel generator of $(\mathcal{G}, \pi, H)$. Let $T_{0}=\left(\begin{array}{cc}I_{H} & 0 \\ \theta_{\Gamma} & I_{M^{\perp}}\end{array}\right) \in B\left(H \oplus M^{\perp}\right)$. Denote $F:=$ $\left\{F_{g}=E \sigma(g) T_{0}^{*}\right\}_{g \in \mathcal{G}}$. By Theorem 2.2, $(F, \widetilde{F})$ is a dual g-Riesz basis pair for $H \oplus M^{\perp}$, which is a dilation of $(A \pi(\mathcal{G}), B \pi(\mathcal{G}))$, where $\widetilde{F}=\left\{\widetilde{F}_{g}:=\widetilde{E} \sigma(g) T_{0}^{-1}\right\}_{g \in \mathcal{G}}$ is the canonical dual gRiesz basis of $F$. Since $\left(E^{\prime}, \widetilde{E}^{\prime}\right)$ is a g-Riesz basis dual pair for $H \oplus N$, which is a dilation of $(A \pi(\mathcal{G}), B \pi(\mathcal{G}))$, for arbitrary $g \in \mathcal{G}$, denote $E_{g}^{\prime}=A \pi(g) \oplus C_{g}, F_{g}=A \pi(g) \oplus C_{g}^{\prime}$, where $C_{g} \in B\left(N, H_{0}\right), C_{g}^{\prime} \in B\left(M^{\perp}, H_{0}\right)$. By the proof of Theorem 1.9, there is an invertible operator $\widetilde{T} \in B\left(M^{\perp}, N\right)$ such that $C_{g}=C_{g}^{\prime} \widetilde{T}^{*}$. Therefore,

$$
F_{g}\left(\begin{array}{cc}
I_{H} & 0 \\
0 & \widetilde{T}^{*}
\end{array}\right)=\left(A \pi(g) \oplus C_{g}^{\prime}\right)\left(\begin{array}{cc}
I_{H} & 0 \\
0 & \widetilde{T}^{*}
\end{array}\right)=E_{g}^{\prime}=E \sigma(g) T^{*} .
$$

And then,

$$
F_{g}\left(\begin{array}{cc}
I_{H} & 0 \\
0 & \widetilde{T}^{*}
\end{array}\right)=E \sigma(g)\left(\begin{array}{cc}
I_{H} & \theta_{\Gamma}^{*} \\
0 & I_{M^{\perp}}
\end{array}\right)\left(\begin{array}{cc}
I_{H} & 0 \\
0 & \widetilde{T}^{*}
\end{array}\right)=E \sigma(g) T^{*} .
$$


Since $E \sigma(\mathcal{G})$ is a g-Riesz basis for $H \oplus M^{\perp}$, it is g-complete. Then

$$
\left(\begin{array}{cc}
I_{H} & \theta_{\Gamma}^{*} \\
0 & I_{M^{\perp}}
\end{array}\right)\left(\begin{array}{cc}
I_{H} & 0 \\
0 & \widetilde{T}^{*}
\end{array}\right)=\left(\begin{array}{cc}
I_{H} & \theta_{\Gamma}^{*} \widetilde{T}^{*} \\
0 & \widetilde{T}^{*}
\end{array}\right)=T^{*}
$$

Hence,

$$
T=\left(\begin{array}{cc}
I_{H} & 0 \\
\widetilde{T} \theta_{\Gamma} & \widetilde{T}
\end{array}\right)
$$

where $T^{\prime}=\widetilde{T} \theta_{\Gamma} \in B(H, N)$.

The following is our main result which shows that if the dilation Riesz generator dual pair of a frame generator alternate dual pair has a relationship with that of another frame generator alternate dual pair by a pair of operators, then one of the corresponding operators is in the form of a lower triangular matrix.

Proposition 2.5 Let $(\mathcal{G}, \pi, H),\left(\mathcal{G}, \pi_{1}, N_{1}\right),\left(\pi_{2}, \mathcal{G}, N_{2}\right)$ be unitary representations of $\mathcal{G}$ on $H, N_{1}, N_{2}$ respectively, $(A, B),\left(A, B^{\prime}\right)$ be frame generator alternate dual pairs of $(\mathcal{G}, \pi, H)$, where $A, B, B^{\prime} \in B\left(H, H_{0}\right)$. Suppose that there exist pairs of dual Riesz generators $\left(E^{\prime}, \widetilde{E}^{\prime}\right)$, $(F, \widetilde{F})$ of $\left(\mathcal{G}, \sigma_{1}, H \oplus N_{1}\right),\left(\mathcal{G}, \sigma_{2}, H \oplus N_{2}\right)$ which are also dilations of $(A, B),\left(A, B^{\prime}\right)$ respectively, where $\sigma_{1}=\pi \oplus \pi_{1}, \sigma_{2}=\pi \oplus \pi_{2}, E^{\prime}, \widetilde{E}^{\prime} \in B\left(H \oplus N_{1}, H_{0}\right), F, \widetilde{F} \in B\left(H \oplus N_{2}, H_{0}\right)$. If there is an invertible operator $T \in B\left(H \oplus N_{1}, H \oplus N_{2}\right)$ such that $F \sigma_{2}(g)=E^{\prime} \sigma_{1}(g) T^{*}$ for any $g \in \mathcal{G}$. Then $T$ is in the form of the following:

$$
T=\left(\begin{array}{cc}
I_{H} & 0 \\
T^{\prime} & \widetilde{T}
\end{array}\right)
$$

where $T^{\prime} \in B\left(H, N_{2}\right), \widetilde{T} \in B\left(N_{1}, N_{2}\right)$.

Proof Let $\Gamma=\widetilde{A}-B \in B\left(H, H_{0}\right), \Gamma^{\prime}=\widetilde{A}-B^{\prime} \in B\left(H, H_{0}\right)$. We can easily examine $\Gamma, \Gamma^{\prime}$ are Bessel generators of $(\mathcal{G}, \pi, H)$ and $\operatorname{ran} \theta_{\Gamma}, \operatorname{ran} \theta_{\Gamma^{\prime}} \subset\left(\operatorname{ran} \theta_{A}\right)^{\perp}$.

Similar to the proof of Theorem 2.3, we can construct

$$
T_{1}=\left(\begin{array}{cc}
I_{H} & 0 \\
0 & \widetilde{T}_{1}
\end{array}\right)\left(\begin{array}{cc}
I_{H} & 0 \\
\theta_{\Gamma} & I_{M^{\perp}}
\end{array}\right)=\left(\begin{array}{cc}
I_{H} & 0 \\
\widetilde{T}_{1} \theta_{\Gamma} & \widetilde{T}_{1}
\end{array}\right) \in B\left(H \oplus M^{\perp}, H \oplus N_{1}\right)
$$

and

$$
T_{2}=\left(\begin{array}{cc}
I_{H} & 0 \\
0 & \widetilde{T}_{2}
\end{array}\right)\left(\begin{array}{cc}
I_{H} & 0 \\
\theta_{\Gamma^{\prime}} & I_{M^{\perp}}
\end{array}\right)=\left(\begin{array}{cc}
I_{H} & 0 \\
\widetilde{T}_{2} \theta_{\Gamma^{\prime}} & \widetilde{T}_{2}
\end{array}\right) \in B\left(H \oplus M^{\perp}, H \oplus N_{2}\right),
$$

where $\widetilde{T}_{1} \in B\left(M^{\perp}, N_{1}\right), \widetilde{T}_{2} \in B\left(M^{\perp}, N_{2}\right)$ are invertible. (In fact, we can let $E^{\prime}=A \oplus C_{1}$, $F=A \oplus C_{2}$, where $C_{1} \in B\left(N_{1}, H_{0}\right), C_{2} \in B\left(N_{2}, H_{0}\right)$. By Theorem 1.9, there are invertible operators $\widetilde{T}_{1} \in B\left(M^{\perp}, N_{1}\right), \widetilde{T}_{2} \in B\left(M^{\perp}, N_{2}\right)$ such that $C_{g}^{1} \widetilde{T}_{1}^{*}=C_{1} \pi_{1}(g)$ and $C_{g}^{2} \widetilde{T}_{2}^{*}=C_{2} \pi_{2}(g)$, where $E_{g}^{1}=E \sigma(g)\left(T_{0}^{1}\right)^{*}, E_{g}^{2}=E \sigma(g)\left(T_{0}^{2}\right)^{*}$ for every $g \in \mathcal{G}, T_{0}^{1}=\left(\begin{array}{cc}I_{H} & 0 \\ \theta_{\Gamma} & I_{M^{\perp}}\end{array}\right), T_{0}^{2}=\left(\begin{array}{cc}I_{H} & 0 \\ \theta_{\Gamma}^{\prime} & I_{M^{\perp}}\end{array}\right) \in$ $B\left(H \oplus M^{\perp}\right)$.) 
Evidently, $T_{1}, T_{2}$ are invertible, $E \sigma(g) T_{1}^{*}=E^{\prime} \sigma_{1}(g), E \sigma(g) T_{2}^{*}=F \sigma_{2}(g)$ for any $g \in \mathcal{G}$.

Denote $T_{1}^{\prime}=\widetilde{T}_{1} \theta_{\Gamma} \in B\left(H, N_{1}\right), T_{2}^{\prime}=\widetilde{T}_{2} \theta_{\Gamma^{\prime}} \in B\left(H, N_{2}\right)$.

Then $F \sigma_{2}(g)=E \sigma(g) T_{2}^{*}=E^{\prime} \sigma_{1}(g)\left(T_{1}^{*}\right)^{-1} T_{2}^{*}$ for any $g \in \mathcal{G}$. Because $T_{2}\left(T_{1}\right)^{-1} \in B(H \oplus$ $N_{1}, H \oplus N_{2}$ ) and

$$
T_{2}\left(T_{1}\right)^{-1}=\left(\begin{array}{cc}
I_{H} & 0 \\
T_{2}^{\prime} & \widetilde{T}_{2}
\end{array}\right)\left(\begin{array}{cc}
I_{H} & 0 \\
-\widetilde{T}_{1}^{-1} T_{1}^{\prime} & \widetilde{T}_{1}^{-1}
\end{array}\right)=\left(\begin{array}{cc}
I_{H} & 0 \\
\widetilde{T}_{2}\left(\theta_{\Gamma^{\prime}}-\theta_{\Gamma}\right) & \widetilde{T}_{2} \widetilde{T}_{1}^{-1}
\end{array}\right) .
$$

Denote $T^{\prime}:=\widetilde{T}_{2}\left(\theta_{\Gamma^{\prime}}-\theta_{\Gamma}\right) \in B\left(H, N_{2}\right), \widetilde{T}=\widetilde{T}_{2} \widetilde{T}_{1}^{-1} \in B\left(N_{1}, N_{2}\right)$.

Since $F \sigma_{2}(g)=E^{\prime} \sigma_{1}(g) T^{*}$ for any $g \in \mathcal{G}$ and $E^{\prime} \sigma_{1}(\mathcal{G})$ is g-complete, it follows that $T=$ $T_{2}\left(T_{1}\right)^{-1}$.

From the above result, we have another illustration that there is an operator which is in the form of a lower triangular matrix to construct a relationship between the dilation Riesz generator pair of a frame generator alternate dual pair and that of another frame generator alternate dual pair.

Corollary 2.6 Let $(\mathcal{G}, \pi, H),\left(\mathcal{G}, \pi_{1}, N_{1}\right),\left(\pi_{2}, \mathcal{G}, N_{2}\right)$ be unitary representations of $\mathcal{G}$ on $H$, $N_{1}, N_{2}$ respectively, $(A, B),\left(A, B^{\prime}\right)$ be frame generator alternate dual pairs of $(\mathcal{G}, \pi, H)$, where $A, B, B^{\prime} \in B\left(H, H_{0}\right)$. Suppose that there exist pairs of dual Riesz generators $\left(E^{\prime}, \widetilde{E}^{\prime}\right),(F, \widetilde{F})$ of $\left(\mathcal{G}, \sigma_{1}, H \oplus N_{1}\right),\left(\mathcal{G}, \sigma_{2}, H \oplus N_{2}\right)$ which are also dilations of $(A, B),\left(A, B^{\prime}\right)$ respectively, where $\sigma_{1}=\pi \oplus \pi_{1}, \sigma_{2}=\pi \oplus \pi_{2}, E^{\prime}, \widetilde{E}^{\prime} \in B\left(H \oplus N_{1}, H_{0}\right), F, \widetilde{F} \in B\left(H \oplus N_{2}, H_{0}\right)$. Then there is an invertible operator $T \in B\left(H \oplus N_{1}, H \oplus N_{2}\right)$ such that $F \sigma_{2}(g)=E^{\prime} \sigma_{1}(g) T^{*}$ for any $g \in \mathcal{G}$, where

$$
T=\left(\begin{array}{cc}
I_{H} & 0 \\
T^{\prime} & \widetilde{T}
\end{array}\right)
$$

$T^{\prime} \in B\left(H, N_{2}\right), \widetilde{T} \in B\left(N_{1}, N_{2}\right)$.

Proof It is direct by the proof of Theorem 2.5.

Acknowledgements

Not applicable.

\section{Funding}

This work is supported by the National Natural Science Foundation of China (Nos. 11671201 and 11771379).

Availability of data and materials

Not applicable.

Competing interests

The authors declare that they have no competing interests.

Authors' contributions

All authors contributed to each part of this work equally and read and approved the final manuscript.

\section{Publisher's Note}

Springer Nature remains neutral with regard to jurisdictional claims in published maps and institutional affiliations. 
References

1. Abdollahpour, M.: Dilation of a family of g-frames. Wavelets Linear Algebra 1, 9-18 (2014)

2. Abdollahpour, M.: Dilation of dual g-frames to dual g-Riesz bases. Banach J. Math. Anal. 9(1), 54-66 (2015)

3. Bishop, S., Heil, C., Koo, Y., Lim, J.: Invariances of frame sequences under perturbations. Linear Algebra Appl. 432(6), 1501-1514 (2010)

4. Christensen, O., Lennard, C., Lewis, C.: Perturbation of frames for a subspace of a Hilbert space. Rocky Mt. J. Math. 30(4), 1237-1249 (2000)

5. Gabardo, J., Han, D.: Frames associated with measurable space. Adv. Comput. Math. 18(2-4), 127-147 (2003)

6. Guo, X:: Wandering operators for unitary systems of Hilbert spaces. Complex Anal. Oper. Theory 10(4), 703-723 (2016)

7. Guo, X.: Similarity and parameterizations of dilations of pairs of dual group frame in Hilbert spaces. Acta Math. Sin Engl. Ser. 33(12), 1671-1683 (2017)

8. Guo, X., Han, D.: Joint similarities and parameterizations for Neimark complementary frames. J. Math. Anal. Appl. 462(1), 148-156 (2018)

9. Han, D., Li, P., Meng, B., Tang, W.: Operator valued frames and structured quantum channels. Sci. China Math. 54(11), 2361-2372 (2011)

10. Kaftal, V., Larson, D., Zhang, S.: Operator-valued frames. Trans. Am. Math. Soc. 361(12), 6349-6385 (2009)

11. Meng, B.: Operator-valued frame generators for group-like unitary systems. Oper. Matrices 7(2), 441-464 (2013)

12. Sun, W.: G-frames and g-Riesz bases. J. Math. Anal. Appl. 322(1), 437-452 (2006)

\section{Submit your manuscript to a SpringerOpen ${ }^{\circ}$ journal and benefit from:}

- Convenient online submission

Rigorous peer review

Open access: articles freely available online

- High visibility within the field

- Retaining the copyright to your article

Submit your next manuscript at $\boldsymbol{~ s p r i n g e r o p e n . c o m ~}$ 